\title{
O VALOR ECONÔMICO DE EXISTÊNCIA DO PARQUE NACIONAL DO JAÚ (AMAZONAS) ${ }^{1}$
}

\author{
Ricardo Felix Santana ${ }^{2}$ \\ José Aroudo Mota ${ }^{3}$
}

\section{INTRODUÇÃO}

O paradigma da proteção dos recursos naturais passou de uma busca da conservação de amostras representativas de ecossistemas - em face do avanço da destruição do ambiente natural pelas exigências do desenvolvimento - para o enfoque principal sobre a conservação/preservação da biodiversidade. Cuidado intensivo e biotecnologia podem preservar alguma diversidade que de outro modo seria perdida. Mas a maior dimensão de tal preservação é demasiadamente pequena se comparada à que pode ou poderia ter sido sustentada em reservas naturais adequadamente projetadas e protegidas (CONWAY, 1997).

Pode-se exemplificar o caso das florestas tropicais que vêm sendo eliminadas com altas taxas de desmatamento, como é observado na Amazônia brasileira. A sociedade deve atribuir valores sociais a essas florestas e, dessa forma, valores econômicos devem ser conferidos aos produtos madeireiros e não-madeireiros, à vida silvestre e a outros benefícios associados à existência da floresta, de forma que a análise de custo-benefício relevante possua uma base mais completa e mais precisa (SHENG, 1997).

1 A pesquisa foi inicialmente publicada como Texto para Discussão $\mathrm{n}^{\circ} 1008$ pelo Instituto de Pesquisa Econômica Aplicada - IPEA. Portanto, esta versão foi resumida a partir do texto original. Agradecemos ao corpo editorial do IPEA pela permissão em publicar este trabalho.

Analista de Ciência e Tecnologia do Conselho Nacional de Desenvolvimento Científico e Tecnológico (CNPq).

3 Pesquisador do Instituto de Pesquisa Econômica Aplicada (IPEA) e Professor do Centro de Desenvolvimento Sustentável (CDS) da Universidade de Brasília (UnB). 
Os pesquisadores dos recursos naturais estão a cada dia mais convencidos da importância da determinação do valor de existência como uma ferramenta para a tomada de decisão sobre a alocação de recursos. Essas e outras questões da política ambiental transformam, em grande parte, a valoração de áreas naturais ou a conservação de espécies por pessoas que não têm nenhum contato direto com determinado recurso, em um fator importante nas tomadas de decisão (LARSON, 1993).

A estimativa do valor de existência é uma ferramenta importante para fundamentar decisões, notadamente de políticas públicas. Existe um argumento econômico para se proteger o meio ambiente, em adição a qualquer questão ética, pois os resultados de políticas públicas ambientais resultam em benefícios para alguns grupos, e em custos, para outros (HANEMANN, 1997; NOGUEIRA e MEDEIROS, 1997; PEARCE, 1993 e BISHOP e WELSH 1992). Para Ehrenfeld (1997), "o valor é uma parte intrínseca da diversidade; não depende das propriedades das espécies em questão, dos usos que se farão ou não de espécies em particular ou do seu alegado papel no equilíbrio dos ecossistemas globais. Para a diversidade biológica o valor existe".

No caso das Unidades de Conservação de uso indireto dos recursos, tem sido difícil a quantificação dos benefícios sociais que essas áreas geram, em razão dos diversos valores nelas existentes. Ainda assim, grandes esforços têm sido empreendidos no sentido de se medir, em termos econômicos, esses benefícios, o que tem sido feito por meio da avaliação de valores indiretos das funções dos ecossistemas, como a proteção de bacias hidrográficas, a regulação do clima e a fotossíntese e proteção de solos. Também são verificados valores intangíveis, tais como manter opções para o futuro ou conhecer e respeitar a existência de outras espécies (BERNARDES, 1999).

\section{CONTEXTUALIZAÇÃO DA PESQUISA}

O Parque Nacional do Jaú (PNJ) é o segundo maior parque do Brasil e o terceiro da América Latina, com área de 2.272 mil hectares, correspondendo a cerca de 1,42\% do Estado do Amazonas. O PNJ localiza-se a aproximadamente $200 \mathrm{~km}$ a noroeste de Manaus. Sua única via de acesso é pelo rio Negro. Uma das peculiaridades mais extraordinárias do PNJ é o fato de ser a única Unidade de Conservação do Brasil que protege quase a totalidade da bacia de um rio extenso, aproximadamente $450 \mathrm{~km}$ do rio Jaú, preservando ecossistemas de águas pretas. O parque está situado no centro de um Corredor Ecológico, apoiado pelo Programa Piloto para a Proteção das Florestas Tropicais do Brasil 
(PPG-7), o da Amazônia Central, que coincide com a Reserva da Biosfera da Amazônia Central.

Esse Parque Nacional foi reconhecido pela Organização das Nações Unidas para Educação, Ciência e Cultura (Unesco) como Patrimônio Mundial Brasileiro em 2001, sendo louvado como uma importante contribuição à sua lista internacional. A Unesco reconhece como Patrimônio Mundial obras e áreas de grande interesse para a história da Terra, ou para a cultura da humanidade. A inclusão de um Sítio na lista de Patrimônio Mundial garante sua proteção e prestígio nacional e internacional, com repercussão direta sobre o afluxo de turistas (BRASIL, 2002).

As distribuições de alguns habitats críticos para fauna e flora aquáticas também variam sistematicamente, de acordo com a área da bacia. As árvores caídas no canal do rio servem como barreiras físicas e são utilizadas como habitat por diversos organismos. Elas são um substrato importante para o crescimento de algas e de vários invertebrados, além de servirem de esconderijo para peixes e outros seres. A variação na freqüência e no tamanho de árvores submersas produz um mosaico complexo de nichos e habitats que abriga uma comunidade diversa de flora e de fauna. A vegetação apresenta predomínio dos tipos florestais densos e abertos, com encraves de formações abertas e savanóides, constituindo aquilo que se convencionou chamar de Áreas de Tensão Ecológica.

\section{OBJETIVOS DA PESQUISA}

Neste estudo, pretende-se avaliar em que medida o valor de existência pode ser utilizado na formulação e na implementação de políticas públicas para a gestão de Unidades de Conservação. A internalização do valor de existência de UC ainda não faz parte da quantificação dos benefícios gerados pela manutenção dos serviços ambientais. Estudos de valoração de Unidades de Conservação vêm sendo incentivados no Brasil, porém o número ainda não alcançou um valor significativo de casos. Como são poucos os estudos de casos para quantificar tal valor de existência, visando à formulação e à implementação de políticas públicas ambientais, o objetivo geral desta pesquisa é avaliar a percepção dos bolsistas de PQ do CNPQ no tocante aos aspectos ecológicos e de valor de existência do Parque Nacional do Jaú. De acordo com a proposição geral, dispõe-se a analisar os seguintes objetivos específicos:

- avaliar, a partir das preferências dos bolsistas de PQ do CNPQ, as suas percepções quanto à preservação do PNJ; e 
- estimar o valor de existência para o PNJ atribuído pelos bolsistas de PQ do CNPQ.

\section{MATERIAIS E MÉTODO}

A população sob amostra é constituída de pesquisadores que possuem bolsa de Produtividade em Pesquisa (PQ) do Conselho Nacional de Desenvolvimento Científico e Tecnológico (CNPQ). Em 2003 foram implementadas aproximadamente 7.775 bolsas de PQ em todas as áreas do conhecimento da tabela do CNPQ (BRASIL, 2003). A distribuição das bolsas pelos estados brasileiros é muito desigual, refletindo a concentração da pesquisa no eixo Sudeste-Sul, com difusão do conhecimento para as regiões mais carentes - Norte, Nordeste e Centro-Oeste.

No presente estudo foi adotada a técnica de amostragem aleatória simples, a qual foi estimada com $3,55 \%$ de erro e $95 \%$ de confiabilidade. Para eliciar-se o valor de existência médio para o PNJ, utilizou-se o Método de Valoração Contingente (MVC), com o auxílio de uma caixa de e-mails.

Com o objetivo de facilitar o procedimento de envio e recebimento dos questionários foi desenvolvido um sistema que executou essas tarefas. As etapas para a coleta de dados estão inseridas em três ambientes com atores distintos: Serviço de Informática do CNPQ, Respondentes (Bolsistas de PQ) e Pesquisador.

$\mathrm{O}$ valor de existência refere-se ao quanto às pessoas valorizam os ativos naturais, independentemente de seus usos, o qual está relacionado com os sentimentos de altruísmo, responsabilidade e atitude das pessoas em relação à manutenção da biodiversidade (MOTA, 2003; NORTON, 1997; PEARCE e TURNER, 1990; RANDALl, 1997; Marques e COMUne, 1996). Além disso, quando eles analisam os verdadeiros mercados, os economistas não estão interessados nos preços em si, mas nos padrões de seleção e nos tipos de preferências que eles implicam (HANEMANN, 1997).

Trabalhos de valoração contingente vêm sendo feitos nos últimos 35 anos, havendo mais de dois mil artigos e estudos relacionados com o tópico. Aplicações ilustrativas desse método para estimar benefícios incluem as seguintes preocupações: melhoria da qualidade do ar e da água; redução do risco de ingestão de água e contaminação de lençóis freáticos; recreação externa; proteção de mangues, áreas desertas, espécies ameaçadas e sítios de herança cultural; melhorias na educação pública e reabilitação de utilidade pública; redução do risco de alimentos e de transporte e assistência à saúde; 
provisão de serviços ambientais básicos, tais como água potável e disposição do lixo em países desenvolvidos.

Considerações sobre o valor de uso-passivo em uma análise econômica foram feitas pelas observações embrionárias de Krutilla, em que muitas pessoas admiram o valor da natureza apenas por ela existir. Krutilla argumenta que essas pessoas obtêm utilidade por meio do prazer vicariante dessas áreas e, como resultado, têm uma positiva disposição para pagar, desde que o governo exerça boa administração sobre a área. Esse valor vem sendo chamado de valor de legado, valor de existência para observação, valor intrínseco, valor inerente, valor de uso-passivo, valor de administração ou valor de não-uso (FREEMAN III, 1993; CARSON, 2000).

\section{ECONOMIA E VALOR DE EXISTÊNCIA}

A imensa diversidade biológica brasileira manifesta-se já na extraordinária riqueza de ecossistemas. No interior de cada bioma brasileiro, entretanto, são identificadas inumeráveis subunidades biogeográficas ou fisiográficas. A diversidade brasileira é a maior do planeta em plantas superiores, peixes de água doce e mamíferos; a segunda em anfíbios; a terceira em aves; e a quinta em répteis.

Ao lado de toda essa riqueza, os problemas são igualmente de grande magnitude. Entretanto, entre os países chamados de megadiversos, o Brasil pertence a uma minoria que se distingue pelo nível de desenvolvimento da pesquisa científica, com um sistema acadêmico e de instituições de pesquisa extenso e consolidado, embora com lacunas.

Todo esse conjunto de ecossistemas desempenha os serviços ecossistêmicos, em que se incluem a ciclagem de nutrientes e materiais, a produção e a depuração da água e do ar, a existência de sítios de reprodução de estoques pesqueiros e muitos outros, reconhecidos hoje pela relevância na preservação e na conservação da natureza. Dentro desse escopo vem ocorrendo um esforço para quantificar o valor dos serviços.

A necessidade de conceituar o valor econômico do meio ambiente, bem como de desenvolver técnicas para estimar esse valor, surge, basicamente, do fato incontestável de que a maioria dos bens e serviços ambientais e das funções providas ao homem pelo ambiente não é transacionada pelo mercado. Pode-se, até mesmo, ponderar que a necessidade de estimar valores para os ativos ambientais atende às necessidades da adoção de medidas que visem à utilização sustentável do recurso. 
Os recursos naturais não são mercadorias. Sendo assim, não têm preços fixados pelos mercados, pois constituem ativos essenciais à preservação da vida de todos os seres. Dessa forma, faz-se necessário compreender o valor que tem o meio ambiente para a sobrevivência das espécies na Terra - os recursos naturais não têm valor nos mercados convencionais.

Como a maioria dos bens e serviços ambientais não é transacionada no mercado convencional, é necessário estimar o valor econômico do meio ambiente e desenvolver técnicas para realizar tal estimativa. A determinação desses valores visa a utilização sustentável dos recursos naturais.

O meio ambiente, ao desempenhar funções imprescindíveis à vida humana, apresenta, em decorrência, valor econômico positivo, mesmo que não refletido diretamente pelo funcionamento do mercado. Portanto, não se pode atribuir valor zero, correndo risco de uso excessivo ou até mesmo de sua completa degradação. Um princípio básico a ser observado é que o ambiente e o sistema econômico interagem, quer por meio dos impactos que o sistema econômico provoca no ambiente, quer pelo impacto que os recursos naturais causam na economia.

Tem-se usado o termo valor de existência de um recurso para definir valor para um recurso natural que é motivado pela fonte interna à função de utilidade do indivíduo, além do uso pessoal. Por que consumidores valoram algo que eles não consomem pessoalmente? Por altruísmo para com parentes e amigos, ou outros que possam ser usuários, para com as futuras gerações de usuários, ou para com animais, que pode motivar valores de existência. Alguns podem argüir que valores de existência para o obscuro e desconhecido são passíveis de serem contemplados em uma prioridade.

Argumenta-se que existem razões que forçam a não-exclusão do valor de existência em alguns casos. No nível da teoria abstrata, pode-se distinguir entre preferências e informações. Quando consumidores expressam suas preferências submetidas a um orçamento, forçando as firmas a maximizar os seus lucros sobre uma dada tecnologia de produção, o conhecimento perfeito é então assumido. Uma maneira de racionalizar a escassa informação acumulada sobre recurso é ignorar o que não seja relevante para a escolha corrente.

Quanto aos valores morais que as pessoas em geral atribuem às espécies estes são bem altos. Respostas a questionários indicam que os entrevistados colocam um valor surpreendentemente alto apenas sobre o conhecimento de que algo exista, independentemente de qualquer uso que possa ocorrer com tal espécie. Economistas, usando um método chamado avaliação contingente, criaram mercados fictícios nos quais eles podem perguntar às pessoas quanto elas estariam dispostas a pagar para proteger 
uma espécie, independentemente das possibilidades de uso dessa espécie. Se valores existenciais podem ser concebidos como um rudimentar indicador de valores morais para os objetivos atuais, podemos dizer que as espécies têm um valor moral consideravelmente alto, mensurável em dólares.

\section{RESULTADOS DA PESQUISA}

O estudo piloto foi realizado em setembro de 2002, para 10\% da totalidade dos bolsistas de Produtividade em Pesquisa (PQ), obtendo-se uma taxa de retorno de $12,34 \%$. A etapa de aplicação do questionário final ocorreu no mês de janeiro de 2003, quando se obteve uma amostra de $9,38 \%$, o que possibilitou grau de confiabilidade de $95 \%$ e $3,55 \%$ de margem de erro.

De acordo com a amostra, 33,3\% dos entrevistados estavam dispostos a pagar pela existência do PNJ, enquanto $66,7 \%$ não apresentaram tal disposição. Desses respondentes, a maior parte era bolsista PQ-2C, o que equivale a $24 \%$ dos respondentes, e o menor número de respondentes era PQ1A $(\operatorname{com} 9,3 \%)$.

A disposição a pagar atribuída pelos bolsistas de Produtividade em Pesquisa do CNPQ, captada pelo método de valoração contingente, foi de $\mathrm{R} \$ 2,12$ per capita por bolsista/mês, o que representa, por ano, $\mathrm{R} \$ 197.796$, e $\mathrm{R} \$ 1$ milhão para todo o sistema de incentivo à pesquisa patrocinado pelo CNPQ. Esses montantes representam valores de benefícios ou excedentes que os pesquisadores do CNPQ se propõem a pagar periodicamente pela existência do PNJ.

A escolha da disposição a pagar ou não, bem como os valores eliciados, que demonstram o comportamento dos respondentes com uma margem de erro de $10 \%$, são explicados por três variáveis: duas socioeconômicas, compostas pela renda familiar e pela idade; e uma referente às atitudes dos respondentes em relação ao PNJ, e se compõe do nível de importância da existência do PNJ.

Em outras palavras, somente essas variáveis são relevantes na explicação da variação da disposição média a pagar. Dos modelos analisados, o $\log$ duplo, ou seja, logaritmo natural nas variáveis dependentes e independentes, revelou-se mais adequado por apresentar os testes clássicos estatísticos mais robustos. Assim, escolheu-se o modelo log-log para explicar as variações ocorridas na disposição a pagar dos bolsistas pesquisadores, o qual tem a seguinte configuração: 


$\begin{array}{lrrrr}L N D a p= & 2,626+0,195 & L N(\text { Renda })+0,404 L N(\text { Idade })+0,374 L N(\text { Existenc }) \\ \text { 't' } & -2,220 & 1,951 & 1,640 & 2,212 \\ \text { 'sig' } & 0,027 & 0,051 & 0,102 & 0,027 \\ \text { 'F' } & 4,491 & & & \\ \text { 'sig' } & 0,004 & & \end{array}$

ONDE: LNDap = Logaritmo natural da disposição a pagar; LN $($ Renda $)=$ Logaritmo natural da renda familiar mensal do respondente; LN (Idade) = Logaritmo natural da idade do respondente; e LN (Existenc) = Logaritmo natural do nível de importância dado à existência do PNJ.

Como os dados foram estratificados por área do conhecimento, a predominância foi a de Ciências Biológicas, com 21,1\% dos respondentes; as áreas do conhecimento menos representativas foram a de Lingüística, Letras e Artes, totalizando 1,9\%. Além disso, os pesquisadores em Ciências Sociais Aplicadas apresentaram a maior média de disposição a pagar, alcançando $\mathrm{R}$ \$ 2,68, enquanto os pesquisadores das Engenharias apresentaram a menor média, $\mathrm{R} \$ 1,28$. Em relação à categoria/nível, encontram-se os pesquisadores $1 \mathrm{~A}$ com a maior média de disposição a pagar, atingindo $R$ \$2,23, contra uma DAP média mínima de $\mathrm{R} \$ 1,68$ para os pesquisadores 2 A (Figura 1).

Como forma de captar a percepção dos respondentes sobre questões ambientais, foram aplicadas algumas questões que abordavam os Parques Nacionais e mais especificamente o Parque Nacional do Jaú. A nota média dada ao nível de importância da existência do PNJ foi 8,75. Essa foi uma das variáveis que explicam a disposição a pagar média no modelo log-log (Figura 2).

Dos respondentes, $66,8 \%$ afirmaram que já haviam visitado um parque nacional, e a disposição a pagar média destes foi de $\mathrm{R} \$ 2,46$, enquanto a dos que não visitaram um parque nacional foi de $\mathrm{R} \$ 1,93$. Quanto às funções dos parques nacionais, 67,4\% indicaram não saber responder essa questão, enquanto $27,9 \%$ indicaram como função principal a conservação dos recursos naturais. Em relação às horas de lazer passadas em um Parque Nacional, 90,7\% dos entrevistados consideram importantes e muito importantes esses momentos.

No que se refere ao grau de responsabilidade pelos problemas ambientais, os resultados amostrais sinalizam que o poder público e a sociedade são os principais responsáveis pelo agravamento da degradação dos recursos da natureza. Quanto aos níveis de responsabilidade os mesmos foram atribuídos 
Figura 1 - DISPOSIÇÃO A PAGAR MÉDIA POR ÁREA DO CONHECIMENTO E PERCENTUAL DE RESPONDENTES

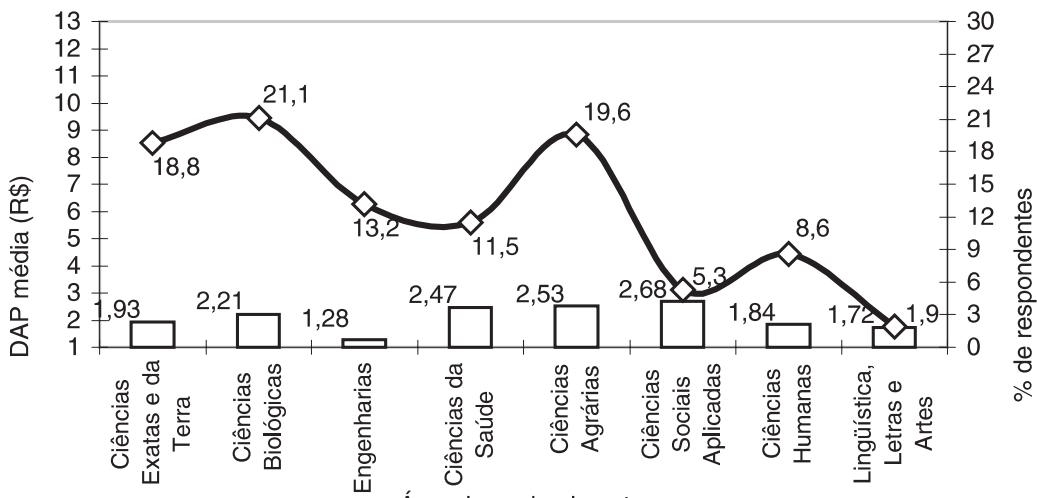

FONTE: Elaboração dos autores.

Figura 2 - GRAU MÉDIO DE IMPORTÂNCIA PELA EXISTÊNCIA DO PNJ CONFORME A ÁREA DO CONHECIMENTO DO PESQUISADO

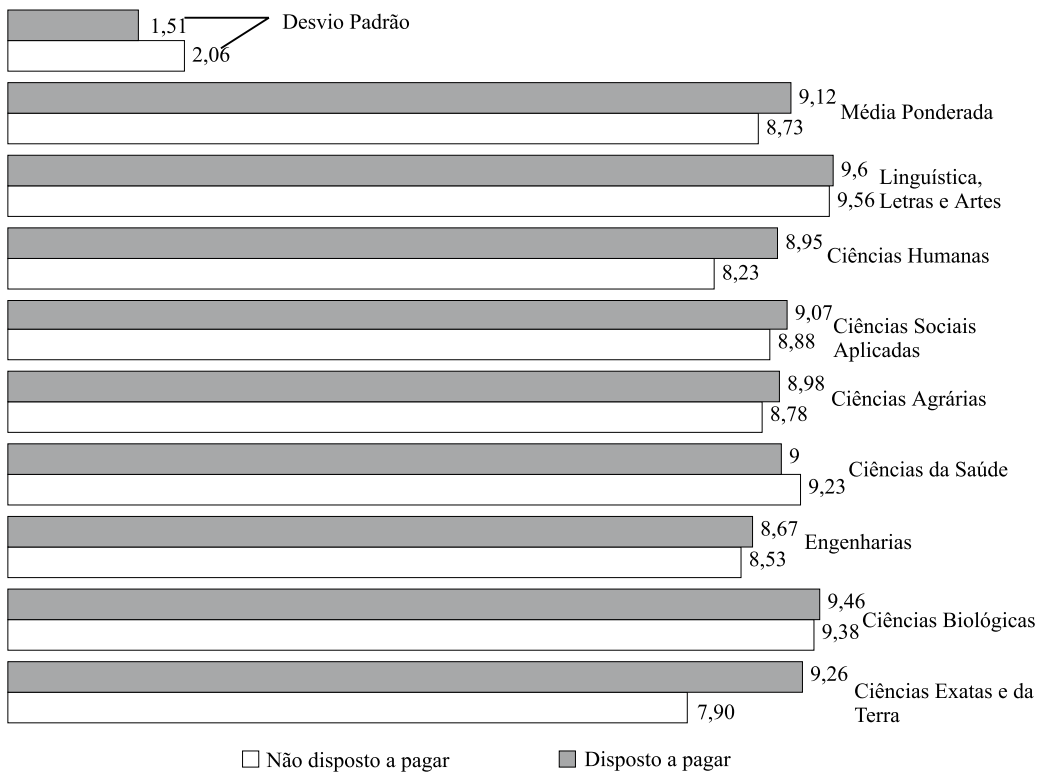

FONTE: Elaboração dos autores 
aos principais problemas relacionados à degradação da natureza, cujo destaque é a expansão da fronteira agrícola, em que 60,5\% dos entrevistados afirmaram que a sociedade é o principal agente de degradação ambiental. Concomitantemente, $53,1 \%$ dos entrevistados indicam que o poder público é o principal responsável pela desigualdade social.

Deve ser ressaltada a relevância ecológica do PNJ, podendo-se afirmar que o conjunto de ambientes que compõem a área de estudo fornece uma série de serviços ambientais para as comunidades locais e regionais. Os benefícios ecológicos prestados por rios, lagos, igarapés e áreas florestais garantem as condições adequadas para a sobrevivência de todas as espécies. Sobre a exploração dos recursos naturais, 98\% declararam ser necessário um controle mais rígido, enquanto apenas $2 \%$ não sabem ou não consideram necessárias alterações nos controles existentes. Desse modo, quanto ao conhecimento das questões ecológicas acordadas pelo Brasil na Eco-92, Conferência realizada no Rio de Janeiro em 1992, 28,3\% dos entrevistados não conhecem os termos da Agenda 21, destacando-se que 15,6\% ignoram as diretrizes da Política Nacional da Biodiversidade. Dos 71,7\% que conhecem os termos da Agenda 21, destaca-se que 42,7\% defendem ser correta a instituição de princípios e diretrizes para a Política Nacional da Biodiversidade.

\section{CONCLUSÕES E RECOMENDAÇÕES}

Esta pesquisa proporcionou avaliar as preferências de um grupo de pesquisadores do CNPQ a partir da aplicação de questionário que objetivava eliciar valores contingentes. Esses valores referem-se às disposições a pagar pela existência do Parque Nacional do Jaú, e são traduzidos em economia ambiental como benefícios ou excedentes gerados em razão da percepção do grupo focal estudado. Os materiais e o método utilizados permitiram:

a) Desenhar um questionário capaz de minimizar os vieses possíveis do método de valoração contingente, levando-se em conta as recomendações do painel National Oceanic and Atmospheric Administration (NOAA);

b) Usar o correio eletrônico, o qual constitui um meio de captação de dados com baixo custo, rapidez no envio dos questionários, eficiência no retorno dos dados e sigilo das respostas dos entrevistados;

c) Incluir diversas questões de psicologia comportamental em relação à eliciação contingente a fim de subsidiar as agendas de políticas ambientais;

d) Avaliar que as atitudes relacionadas a comportamentos ambientais podem ser definidas nas funções de utilidade. As atitudes captadas 
nos questionários ajudam a explicar por que determinados valores de disposição a pagar foram explicitados. Questões éticas e morais são incorporadas às avaliações quando levam em conta essas atitudes. Além dos resultados aqui alcançados, a eliciação de valores contingentes é útil na orientação da formulação de políticas públicas setoriais, tais como:

- Diferenciação de escolhas entre grupos: a taxa de respondentes, de acordo com categoria/nível da bolsa de PQ, segue a distribuição do número total de bolsas dentro de cada categoria/nível. Quanto à área do conhecimento, a maior taxa de resposta de bolsistas foi das Ciências Biológicas, seguida pelos bolsistas de Ciências Agrárias e das Ciências Exatas e da Terra. Identificou-se maior preocupação desses pesquisadores com questões relacionadas à gestão de áreas protegidas - o que não cria um estigma -, pois deve ocorrer por influência da formação técnica e do objeto de pesquisa dos pesquisadores. Entretanto, quando se observam os valores de DAP médio de acordo com a categoria/nível e a área do conhecimento, depara-se com resultados diferentes. Considerando-se a categoria/nível, os pesquisadores $1 \mathrm{~A}$ apresentam a maior DAP média, o que deve ser influenciado pela idade dos respondentes;

- Eliciação distinta por área do conhecimento: em relação a este aspecto, encontram-se os pesquisadores de Ciências Agrárias e de Ciências Sociais Aplicadas com os maiores valores de DAP médio. Esse resultado dá maior destaque à importância atribuída pelos pesquisadores de Ciências Agrárias. Nota-se uma taxa elevada de respondentes que não estavam dispostos a pagar pela existência do PNJ, a qual alcançou 66,7\%. Tal resultado foi influenciado pela forma de pagamento criada, que previa o desconto da bolsa de PQ. Alia-se a essa questão a percepção de que se pagam muitos impostos e de que ocorre um baixo retorno na forma de serviços públicos;

- Variáveis comportamentais ajudam na eliciação de valores contingentes: as questões relacionadas com a percepção ambiental dos bolsistas de PQ geraram uma das variáveis que respondem à disposição a pagar pela existência do PNJ, que é o nível de importância da existência do PNJ. O valor médio encontrado, de 8,75 , demonstra o grau de compromisso dos entrevistados em relação à existência do Parque. Outras questões relacionadas com a percepção ambiental dos bolsistas de PQ também têm importância em uma avaliação qualitativa das variáveis;

- Percepção global quanto aos problemas ambientais: uma outra linha de perguntas sobre a percepção ambiental dos respondentes abordou pontos da atualidade e de espectro nacional. Quando se observa que a sociedade em geral e o governo federal foram apontados como os principais responsáveis 
pelos problemas ambientais brasileiros, isso reflete as ações de ocupação dos espaços e o uso dos recursos por diversas atividades produtivas relacionadas à existência humana, bem como a descrença dos brasileiros quando se fala das instituições públicas de todas as áreas. Essa afirmação é reforçada quando se verifica que $98,47 \%$ dos respondentes acham necessário um controle mais rígido na exploração dos recursos naturais;

- Disseminação da informação ambiental: a abordagem de políticas públicas ambientais foi avaliada a partir de questões como a Agenda 21 e o acesso à biodiversidade. Percebeu-se que a Agenda 21 é conhecida, pelo menos em parte, pela maioria dos respondentes, a qual considera correta em parte a política de acesso à biodiversidade. Junte-se a esses dados a indicação do desmatamento e da desigualdade social como os principais problemas ambientais existentes no Brasil atualmente, o que demonstra a percepção que os respondentes têm em relacionar, em grande parte, problemas ambientais com problemas sociais; bem como a tendência a afirmar que a solução de um está ligada à solução do outro, em um processo indissociável;

- Nível da informação esperada: quanto ao perfil dos respondentes, os resultados obtidos refletem a população que foi escolhida para a amostragem, que se constitui de bolsistas de PQ do CNPQ, que possuem o nível de doutorado e, em sua maioria, são professores universitários de instituições públicas. Trata-se, portanto, de componentes de uma pequena minoria da população nacional que tem o acesso a informações totalmente diferenciado da grande maioria dos brasileiros. Apesar disso, as suas percepções devem ser levadas em consideração em decorrência da capacidade de criar discussão e fatos que levam à inserção de temas em agendas políticas, nas diversas áreas do conhecimento;

- Eficiência no sistema de coleta de dados: a utilização do correio eletrônico como instrumento para a aplicação de questionários para as pesquisas de valoração contingente deve ser recomendada para futuras pesquisas de valores econômicos de ativos naturais como forma de se criar parâmetros para a formulação de políticas públicas. Os baixos custos de aplicação e a existência de sistemas de informática nos principais órgãos do governo federal, tais como o CNPQ, o Ipea, o Ministério do Meio Ambiente (MMA) e o Instituto Brasileiro do Meio Ambiente e dos Recursos Naturais Renováveis (Ibama), justificam essa recomendação;

- Eliciação de valores complementares: os resultados são úteis para futuras pesquisas. Por isso, recomenda-se o levantamento dos demais valores econômicos para o Parque Nacional do Jaú. O valor de uso deverá ser eliciado tanto pelo uso recreativo como pelo uso dos recursos pelas comunidades tradicionais que residem dentro dos limites do Parque. Assim, o método de 
valoração contingente deverá ser aplicado nas comunidades locais como forma de complementar a composição do valor de existência e de uso.

Mesmo com os resultados alcançados pela pesquisa, recomendamse diversas observações quanto ao uso do método de valoração contingente, destacando-se: $i$ ) a avaliação de vieses do método; $i i)$ a análise do grupo focal a ser estudado; iii) a limitação do suposto valor estimado, o qual restringe somente as preferências assumidas pelo grupo avaliado; e $i v$ ) o desenho de um questionário apropriado para a eliciação das proposições da pesquisa planejada.

\section{RESUMO}

Nas últimas décadas, a discussão sobre a utilização do Valor de Existência (VE) na composição do Valor Econômico Total (VET) tem acentuado a necessidade da sua aplicação na formulação de políticas públicas ambientais. $\mathrm{O}$ seu uso compreende a avaliação de programas e projetos que incluem a busca do desenvolvimento sustentável. Políticas ambientais resultam em benefícios para alguns e em custos para outros. Freqüentemente, os custos de um programa na área ambiental são custos para a sociedade envolvida no programa. No caso das Unidades de Conservação (UC) de uso indireto dos recursos, tem sido difícil a quantificação dos benefícios sociais que essas áreas geram em razão dos diversos valores nelas existentes. Nesses casos, faz-se a avaliação de valores indiretos das funções dos ecossistemas. No presente estudo, foi empregado o Método de Valoração Contingente (MVC), por intermédio da aplicação de um questionário (survey) por meio eletrônico (e-mail). Como contexto, foi utilizado o Parque Nacional do Jaú (PNJ), e a população amostrada foi constituída da totalidade dos bolsistas em Produtividade em Pesquisa (PQ) do Conselho Nacional de Desenvolvimento Científico e Tecnológico (CNPq), os quais declararam uma Disposição a Pagar média no valor de $\mathrm{R} \$ 2,12$. Também foram avaliadas questões sobre percepção ambiental, conhecimento sobre os Parques Nacionais e dados socioeconômicos.

Palavras-chave: Parque Nacional do Jaú, valor de existência, valor econômico total, Método de Valoração Contingente, políticas ambientais. 


\begin{abstract}
In the last decades the quarrel on the use of the Existence Value (EV) in the composition of Total Economic Value (TEV) is accenting the necessity of its application in the formularization of environment public policies. Its use goes since the evaluation of programs and projects until the search of the sustainable development. Environment policies result in benefits for some and costs for others. Frequently, the costs of one program in the environmental a area are costs for the involved society in the program. In the case of the Conservation Units of indirect use of the resources, it has been difficult the quantification of the social benefits that these areas generate in reason of the diverse existing values. In these cases one becomes through the evaluation of indirect values of the functions of ecosystems. In the present study Method of Contingent Valuation was used (MCV), through the one application survey for half electronic (e-mail). As context was used the National Park of Jaú (NPJ), and the showed population was constituted of the totality of the scholarship holders in Productivity in Research (PR) of the National Council for Scientific and Technological Development (CNPq). The PR scholarship holders had arrived at an average Willing to Pay-WTP the value of R $\$ 2,12$. Also partner-economic questions on ambient perception, knowledge on the National Parks and socio-economic data had been made. Key-words: National Park of Jaú, existence value, total economic value, Contingent Valuation Method, environmental policies.
\end{abstract}

\title{
REFERÊNCIAS
}

BERNARDES, Ângela Tresinari. Valores socioculturais de unidades de conservação: herança natural e cultural do homem. Brasília, 1999. Mimeografado.

BISHOP, Richard C.; WELSH, Michael P. Existence values in benefit-cost analysis and damage assessment. Land Economics, n. 68, v. 4, p. 405-417, 1992.

BRASIL. Conselho Nacional de Desenvolvimento Científico e Tecnológico - CNPq. Dados estatísticos. Disponível em: <www.cnpq.br〉. Acesso em: 21 fev. 2003. Brasília, 2003.

BRASIL. Ministério do Meio Ambiente. Secretaria de Biodiversidade e Florestas. Biodiversidade e florestas do Brasil. Brasília: MMA/SBF, 2002.

CARSON, Richard T. Contingent valuation: a user's guide. Environmental, Science and Technology, v. 34, p. 1413-1418, 2000. 
CONWAY, William. A tecnologia pode ajudar na preservação das espécies? In: WILSON, E. O. Biodiversidade. Rio de Janeiro: Nova Fronteira, 1997.

EHRENFELD, D. Por que atribuir um valor à biodiversidade. In: WILSON, E. O. (Org.). Biodiversidade. Rio de Janeiro: Nova Fronteira, 1997.

FREEMAN III, A. Myrick. Nonuse values in natural resource damage assesment. In: KOOP, Raymond J.; SMITH, V. Kerry. (Eds.). Valuing natural assets: the economics of natural resource damage. Washington: Resources for the Future, 1993.

HANEMANN, W. Michael. Economia e preservação da biodiversidade. In: WILSON, E. O. Biodiversidade. Rio de Janeiro: Nova Fronteira, 1997.

LARSON, Douglas M. On measuring existence value. Land Economics, n. 69, v. 4, p. 377-388, Nov. 1993.

MARQUES, João Fernando; COMUNE, Antônio Evaldo. A teoria neoclássica e a valoração ambiental. In: ROMEIRO, Ademar Ribeiro; REYDON, Bastiaan Philip; LEONARDI, Maria Lucia Azevedo. Economia do meio ambiente: teoria, políticas e a gestão de espaços regionais. São Paulo: Unicamp, 1996.

MOTA, José Aroudo. Valorização de recursos naturais: expandindo as fronteiras econômicas, restringindo as fronteiras ambientais. In: NASCIMENTO, Elimar Pinheiro do; DRUMMOND, José Augusto. Amazônia: dinamismo econômico e conservação ambiental. Rio de Janeiro: Garamond, 2003.

NOGUEIRA, Jorge Madeira; MEDEIROS, Marcelino Antonio Asano de. Quanto vale aquilo que não tem valor? Valor de existência, economia e meio ambiente. In: ENCONTRO NACIONAL DE ECONOMIA, 25., 1997, Recife. Anais... Recife: Anpec, v. 2, p. 861-879, 1997.

NORTON, Bryan. Mercadorias, comodidade e moralidade. Os limites da quantificação na avaliação da biodiversidade. In: WILSON, E. O. Biodiversidade. Rio de Janeiro: Nova Fronteira, 1997.

PEARCE, David. Economic values and the natural world. Londres: Earthscan Publication, 1993.

PEARCE, David; TURNER, R. Kerry. Economics of natural resources and the environment. Baltimore: The John Hopkins University Press, 1990.

RANDALL, Alan. O que os economistas tradicionais têm a dizer sobre o valor da biodiversidade. In: WILSON, E. O. Biodiversidade. Rio de Janeiro: Nova Fronteira, 1997.

SHENG, Fulai. Valores em mudança e construção de uma sociedade sustentável. In: CAVALCANTE, Clovis. (Org.). Meio ambiente, desenvolvimento sustentável e políticas públicas. Recife: Fundação Joaquim Nabuco, 1997. 